\title{
Correction to: Maximal orders of global dimension and Krull dimension two
}

\author{
M. Artin
}

Massachusetts Institute of Technology, Department of Mathematics, Cambridge, MA 02139, USA

The last section of the paper [1] contains an error: the auxiliary algebra $A$ which is constructed in the first paragraph is assumed to be a maximal order, which need not be true. So Theorem (6.1) of [1] is incorrect unless the hypothesis that $A$ is maximal is added, and there are some more maximal orders of finite representation type. We have completed their classification in [2], using the methods of [1].

Also, the auxiliary Theorem (1.1) of Brown and Hajarnavis [3] is quoted incorrectly. Contrary to what is stated in section one, it requires the assumption that all simple modules have the same projective dimension. Since our rings have dimension 2, this makes no difference to the paper.

We are indebted to $M$. van den Bergh and C. Hajarnavis for drawing these errors to our attention.

1. Artin, M.: Maximal orders of global dimension and Krull dimension two. Invent. Math. 84, 195-222 (1986)

2. Artin, M., Two-dimensional orders of finite representation type. Manuscr. Math. (in press) (1987)

3. Brown, K.A., Hajarnavis, C.: Homologically homogeneous rings. Trans. Am. Math Soc.. 281, 197-208 (1985) 